\title{
Adaptive Internal Model Control Scheme for a Pneumatic Artificial Muscle
}

\author{
George Andrikopoulos, George Nikolakopoulos, Stamatis Manesis
}

\begin{abstract}
In this article, the model identification and control problem of a Pneumatic Artificial Muscle (PAM) is being considered. The identification of the PAM's model parameters is being carried out by a Recursive Least Square (RLS) based algorithm, while an Internal Model Control (IMC) structure is being synthesized. Experimental studies are being utilized to prove the overall efficiency of the suggested control scheme, regarding: a) set-point tracking performance through selected positioning scenarios, b) adaptability through hysteresis shift compensation and c) robustness through disturbance cancellation.
\end{abstract}

\section{INTRODUCTION}

$\mathrm{T}$ HE McKibben Pneumatic Artificial Muscle (PAM) [1], [2], is a highly nonlinear actuator that, when pressurized, decreases in actuating length. Invented in 1950s by Joseph L. McKibben, the PAM was first utilized as an orthotic appliance for polio patients [3]. The Fluidic Muscle [4], [5], a PAM like the one presented in Fig. 1, differs from the classic McKibben PAM as it utilizes a combined integration of the inner tube and external shell to a single aramidneoprene mesh.

PAM possesses similar properties with those of the organic muscle, combined with several advantages as the ability to provide high power outputs, with relatively light weights and inherent compliance. These characteristics are turning the PAM into a promising actuator choice in anthropomatic technologies, while a variety of such applications have already been appeared in the literature [6], [7]. Moreover, PAM meets the need for safety, simplicity and lightness that human-robot interaction requires and justifies its expanding utilization in medical and biorobotic applications [7].

Control design for PAM-actuated high-performance positioning systems presents difficulties due to the PAM's highly nonlinear nature. Some of the most significant control design approaches for PAM have been developed during the past fifteen years. Those control schemes have included a control based training algorithm on neural networks [8], adaptive position control [9], variable structure control [10], gain scheduling model-based control [11], fuzzy control [12], [13], nonlinear optimal predictive control [14], direct

George Andrikopoulos and Stamatis Manesis are with the Electrical and Computer Engineering Department, University of Patras, GR-26500 Rio, Achaia, Greece (e-mail: \{andrikopg, stam.manesis\} @ece.upatras.gr).

George Nikolakopoulos is with the Control Engineering Group, Department of Computer Science, Electrical and Space Engineering, Luleå University of Technology, SE-97187 Luleå, Sweden (e-mail: geonik@1tu.se). continuous-time adaptive control [15], neuro-fuzzy PID control [16], neural network nonlinear PID control [17], sliding-mode control [18], chattering-free robust variable structure controller [19], hybrid distributed macro-mini control [20], proxy sliding mode control [21] and multiparametric constrained optimal control [22].

The novelty in this article stems from: a) the utilization of an PAM based adaptive Recursive Least Square Internal Model Control scheme, and b) its experimental evaluation. To the authors' best knowledge this is the first time that such a control scheme has been proposed and experimentally evaluated for providing a robust adaptive solution to the positioning problem, in the PAM research literature.

In process control applications, model based control structures are often used to achieve reference tracking and disturbance cancelation. The principle philosophy behind IMC is the statement that "control can be achieved only if the control system encapsulates, either implicitly or explicitly, some representation of the process to be controlled" [23]. Among the advantageous properties of IMC structure is the provision of a transparent and easy framework for the design and tuning of various types of control, its robust behavior through disturbance cancelation during set-point tracking performance, as well as the low complexity and computational cost of its overall synthesis and application, as compared to modern optimal controllers. Furthermore, IMC is known for its adaptation to various input-output system forms, and for its general role in

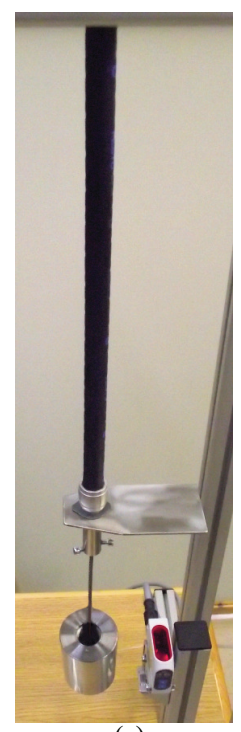

(a)

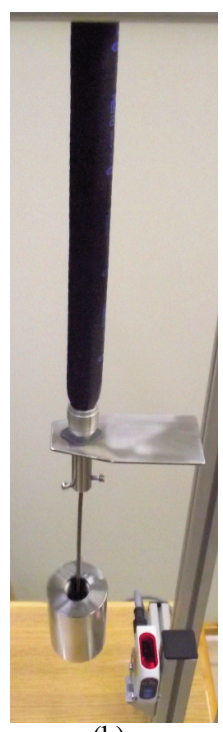

(b)
Figure 1. Pneumatic Artificial Muscle in its (a) relaxed and (b) contracted state, while attached to an external load. 
determining the fundamental requirements associated with feedback control [24], [25].

Similar to open loop control, the main disadvantage of the IMC structure, compared to standard feedback controllers, is the fact that the IM-Controller does not support open loop unstable system integration. That disadvantage is not being encountered in the case of PAM control, considering PAM system's inherent stable nature [2].

In cases of model uncertainty, the IMC is usually detuned to assure stability [26]. The use of the RLS algorithm results in an adaptive model for the PAM actuator, thus, minimizing model uncertainty [27] and omitting the need for the utilization of a detuning filtering process.

The article is organized as follows. In Section 2, the internal model control structure is being presented. In Section 3, multiple experimental studies are being presented to prove the efficacy of the IMC in the positioning problem of a PAM, while evaluating its overall performance and adaptability. Conclusions are being drawn in Section 4.

\section{INTERNAL MODEL CONTROL SCHEME}

In this section, the fundamental components that formulate the proposed IMC scheme are being presented in detail, while the control structure is displayed in Fig. 2.

\section{A. Model Identification}

For the purpose of this article, the PAM actuator is being approximated by an Auto Regressive Moving Average (ARMA) model that is described by:

$$
A(z) \tilde{x}(n)=B(z) P(n-k)
$$

where $\tilde{x}(n)$ is the estimated PAM's displacement from its relaxed position, $P(n)$ is the pressure of the air supplied into the PAM and $k \in Z^{+}$is the system delay. $A(z)$ and $B(z)$ are polynomials with respect to the backward shift operator $z^{-1}$ and defined by the following equations:

$$
A(z)=1+\sum_{i=1}^{n_{a}} a_{i} z^{-i}, \quad B(z)=\sum_{i=1}^{n_{b}} b_{i-1} z^{-(i-1)}
$$

where $n_{a}, n_{b} \in R$ are the maximum orders of the denominator and numerator respectively. The corresponding identification error is being defined by:

$$
e(n)=x_{d}(n)-\tilde{x}(n)
$$

As it has been depicted in Fig. 2, $x_{\mathrm{d}}(n)$ is the real system output $x(n)$ corrupted by additive output disturbance signal $d(n)$, and $\tilde{x}(n)$ is the predicted response of the ARMA model.

For the purposes of this article, the system identification is being carried out via the RLS algorithm [27]. The goal of this adaptive algorithm is to minimize the modified and more

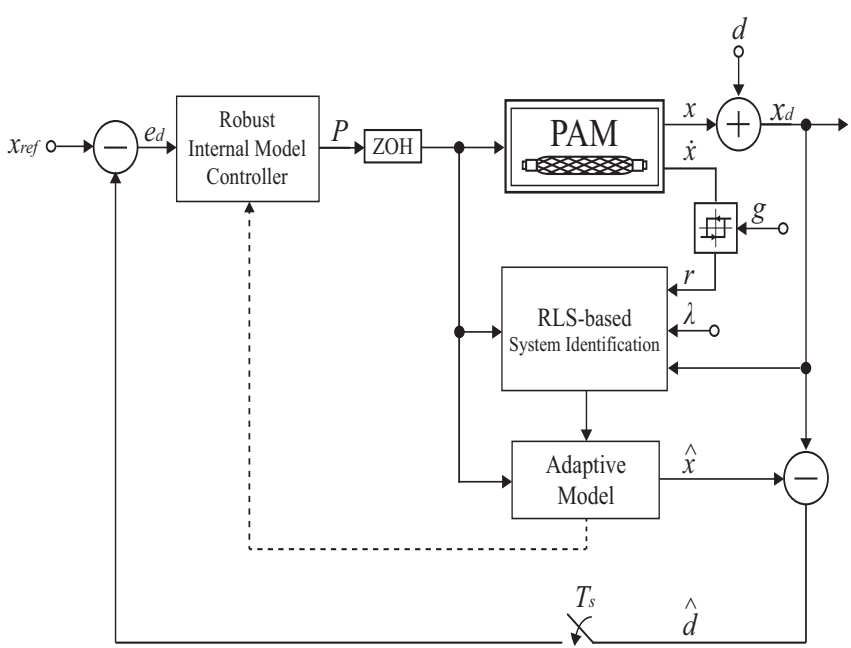

Figure 2. Robust Adaptive Internal Model Control Structure

robust cost function (4), which utilizes the previous $N$ error terms, by appropriately adjusting a parametric vector $\vec{w}(n)$, which contains the unknown ARMA coefficients.

$$
J(n)=E\left[e^{2}(n)\right] \cong \frac{1}{N} \sum_{i=0}^{N-1} e^{2}(n-i)
$$

For improving the identification process, the RLS algorithm was modified further to adjust to the hysteretic nature of PAMs. Specifically, intense hysteretic phenomena alter the PAM dynamics during inflation and deflation operating states. An experimental insight to the hysteresis phenomenon, regarding the PAM utilized in this article, is presented in the next Section.

To account for these alterations and improve the convergence rate and adaptability of the aforementioned algorithm during the operating state transitions, the algorithm was properly modified in order to restart the recursive process in the case of an operating state change. Monitoring of operating changes has been achieved by the signal $r$, which changes from 1 to -1 for inflation and deflation, respectively, and is being denoted as:

$$
r(k)=\left\{\begin{array}{ccc}
1 & \text { for } & \dot{x}(k) \geq g \\
r(k-1) & \text { for } & |\dot{x}(k)|<g \\
-1 & \text { for } & \dot{x}(k) \leq-g
\end{array}\right.
$$

where $g$ is an empirical fine-tuned constant, derived from extended experimental trials by taking into account the measurement accuracy of the laser distance sensor [22]. The purpose of the Schmitt-trigger based filter described in (5) is to avoid undesirable switching during convergence phases when $\dot{x}$ becomes small and because of the additive measurement noise due to finite accuracy of the laser distance sensor.

By taking all the above into consideration, the modified RLS algorithm was formulated and is presented in Table 1. 
TABLE I

MODIFIED RLS ALGORITHM

\begin{tabular}{l} 
Initialization Phase \\
\hline 1. Parameter vector $\vec{w}(n): \vec{w}(0)=\left[\begin{array}{llll}\varepsilon & \varepsilon & \ldots & \varepsilon\end{array}\right]^{T}$, \\
where $\varepsilon$ is a small positive integer. \\
2. Data vector $\vec{\varphi}(n): \vec{\varphi}(0)=\left[\begin{array}{llll}0 & 0 & \ldots & 0\end{array}\right]^{T}$. \\
3. $n \times n$ matrix $L(n): L(0)=\left[\begin{array}{lll}\varepsilon & 0 & 0 \\
0 & \ldots & 0 \\
0 & 0 & \varepsilon\end{array}\right]$ \\
4. Operating state $r(n): r(0)=1$
\end{tabular}

Recursive Computation Phase

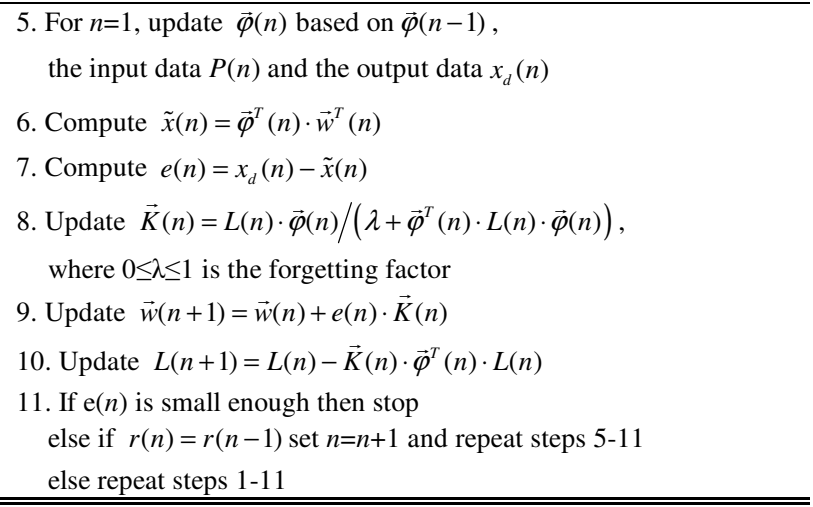

\section{B. Internal Model Controller Synthesis}

Given the PAM model of (1), factorization is performed and the model is separated as shown in (6):

$$
\tilde{G}_{P A M}(z)=\tilde{G}_{P A M}^{+}(z) \tilde{G}_{P A M}^{-}(z)
$$

where $\tilde{G}_{P A M}(z)=B(z) / A(z), \quad \tilde{G}_{P A M}^{+}(z)$ contains the noninvertible and non-minimum phase elements of the model, and $\tilde{G}_{P A M}^{-}(z)$ contains the invertible and minimum phase elements.

The IM-Controller is formulated as it follows:

$$
G_{I M C}(z)=\frac{1}{\tilde{G}_{P A M}^{-}(z)}
$$

Controller complexity, concerning the IMC design procedure [28], is determined by the adaptive model's complexity and the designer's performance requirements. In the IMC strategy, the sensitivity function is provided by [23]:

$$
\varepsilon(z)=\frac{1-G_{I M C}(z) \tilde{G}_{P A M}(z)}{1+G_{I M C}(z)\left[G_{P A M}(z)-\tilde{G}_{P A M}(z)\right]}
$$

Supposing that $\tilde{G}_{P A M}(z)=G_{P A M}(z)$, then

$$
\begin{gathered}
\tilde{\varepsilon}(z)=1-G_{I M C}(z) \tilde{G}_{P A M}(z) \\
\tilde{\eta}(z)=G_{I M C}(z) \tilde{G}_{P A M}(z)
\end{gathered}
$$

The linear appearance of the IM-Controller in the sensitivity function $\tilde{\varepsilon}(z)$, which determines performance, and the complementary sensitivity function $\tilde{\eta}(z)$, which determines robustness, shows the provision of a simpler control framework for robust control system design, as compared to the conventional feedback control procedure.

Consequently, the IM-Controller shows inherent robustness, managing disturbance cancellation and compensating for model uncertainty. Moreover, perfect control is theoretically possible when the controller has been synthesized based on the exact model representation of the process. In this case, the IMC structure has the conceptual advantage of allowing greater concentration on the control design, without concern for control system stability.

In cases of model uncertainty and mismatch between the plant and the process model, the IMC is usually augmented with a low pass filter to ensure that the controller is proper and minimize discrepancies that typically occur at high frequencies. The use of a recursive identification algorithm minimizes model uncertainty [27], thus omitting the need for the utilization of a detuning filtering process. In addition, by appropriately selecting the orders of the ARMA model parameters, and by taking into consideration the inherent overall stability and causality of the PAM system, which recursively leads to absence of right half-plane zeros, no additional filtering is being required to ensure that the IMController is stable, causal and proper.

\section{EXPERIMENTAL SETUP AND CONTROL SCHEME EVALUATION}

\section{A. Experimental Setup}

The experimental setup that has been utilized for the evaluation of the IM-Controller is depicted in Fig. 3. As the test PAM, a Festo DMSP-10-305N-AM-CM Fluidic Muscle was selected, with $10 \mathrm{~mm}$ of internal diameter, $305 \mathrm{~mm}$ of nominal length, operating pressure range between 0 and 8 bar, and motion range between 0 and $0.0733 \mathrm{~m}$.

Additionally, two Festo DMSP-40-305N-AM-CM Fluidic Muscles, with $40 \mathrm{~mm}$ of diameter and $305 \mathrm{~mm}$ of nominal length, are clamped together and connected with the test PAM via a pulley, forming an antagonistic configuration. All three PAMs are on the vertical position with their upper end clumped. The additional pair of PAMs are supplied with air by the same pressure regulator and their purpose is to act as a powerful disturbance generator, bringing the test PAM to its dynamic limits.

Also, two Festo VPPM-8L-L-1-G14-0L10H-V1N-S1 Proportional Pressure Regulators was used to regulate the supply and pressure of the compressed air supplied into the PAMs. A Festo SOEL-RTD-Q50-PP-S-7L laser distance sensor has been utilized to measure the under study PAM's displacement in the vertical axis. A Zemic H3-C3-300kg alloy steel S-type load cell was used to measure the force exerted from the antagonistic operation of the PAMs. The control of the setup operation, as well as the data acquisition, 


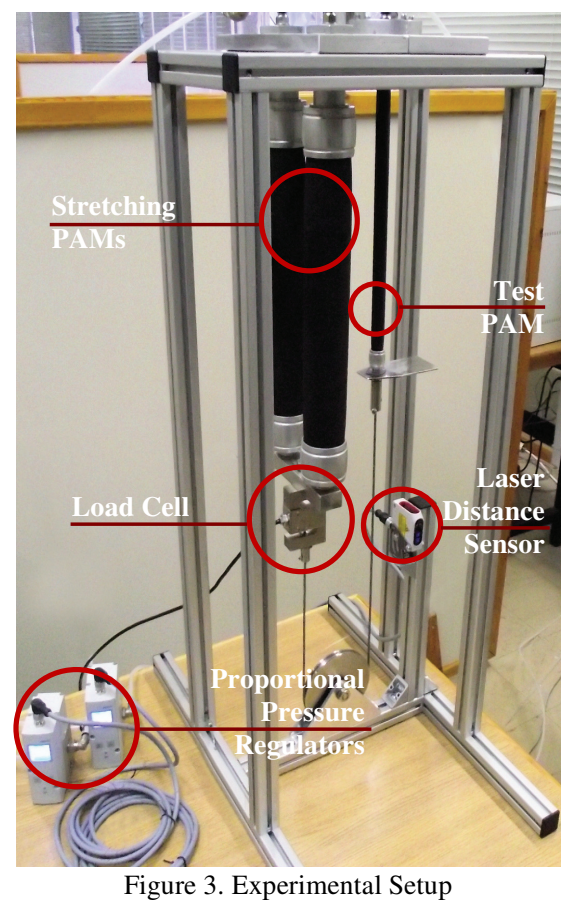

was achieved by utilizing a National Instruments USB-6215 Data Acquisition Card while the setup's programming is carried out in National Instruments LabVIEW.

\section{B. Control Scheme Evaluation}

For the evaluation of the IMC scheme's performance, multiple experimental scenarios were performed. Regarding the optimal ARMA parameter orders of the adaptive model, various experimental trials showed that order combinations other than $n_{a}=2$ and $n_{b}=1$ worsened the control effort's performance and intensified the transient phenomena of the overall response attributes. Thus, for the experiments described below, the orders of the adaptive model were set to $n_{a}=2$ and $n_{b}=1$, the forgetting factor $\lambda$ of the RLS-based algorithm was set to $\lambda=0.99$ and the experimentally finetuned constant $g$ was set to $g=0.005 \mathrm{~m} / \mathrm{s}$.

1) Set-point Tracking Performance: The initial goal of the IMC scheme was to achieve set-point tracking. For the purposes of this experiment, the test-PAM was attached to an external load of approximately $2 \mathrm{~kg}$, as shown in Fig. 1, in order to observe the performance of the scheme while the actuator is under constant load and to avoid horizontal material oscillations during sudden changes in the control effort as a result of the increased elasticity of the PAM.

Multiple set-point experiments were performed and the results are presented in Fig. 4, where the multiple reference signals $x_{\text {ref }}$ are displayed with red dotted lines and the experimental displacement $x$ responses are displayed with black solid lines.

The obtained experimental responses are characterized by smooth and fast convergence, with rising times below $1 \mathrm{sec}$, absence of major oscillations and overshoots, as well as small mean steady state errors that range between 0.1 and $0.5 \%$. The IM-Controller's performance in the reference

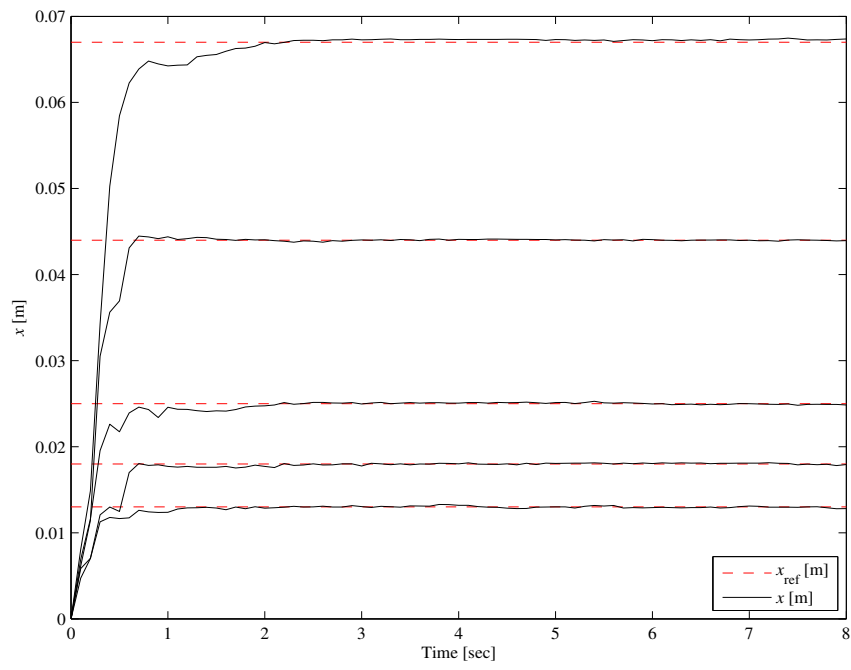

Figure 4. IM-Controller performance during multiple displacement $x$ setpoint tracking responses of the test-PAM.
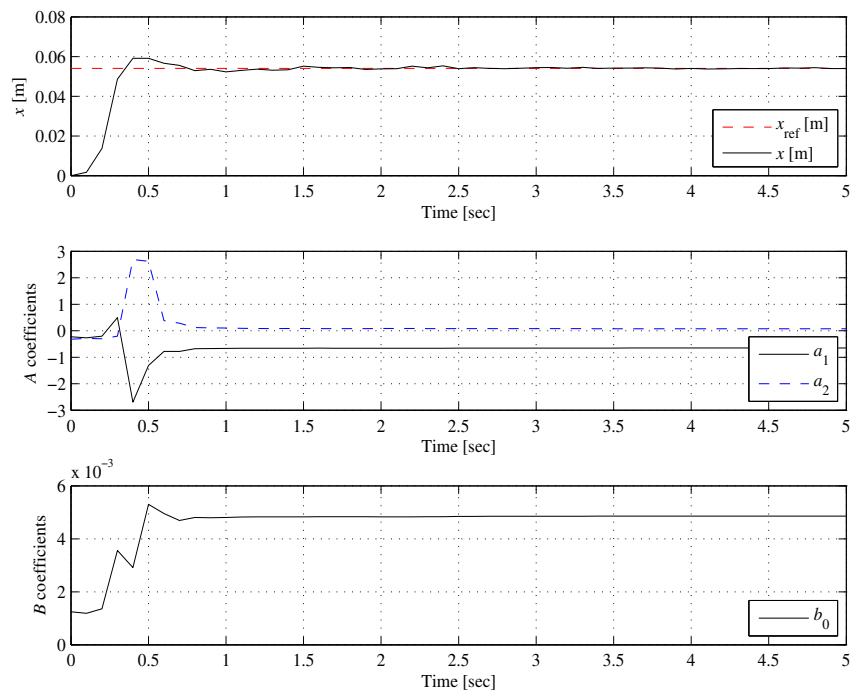

Figure 5. ARMA parameter convergence during a set-point tracking experiment with $x_{\text {initial }}=0$ and $x_{\text {ref }}=0.054 \mathrm{~m}$.

tracking problem is considered successful throughout the motion range of the test-PAM.

2) Model Parameter Convergence: Fig. 5 shows the ARMA parameter convergence of the PAM's adaptive model during a set-point tracking experiment with $x_{\mathrm{ref}}=0.054 \mathrm{~m}$, while being under a constant load of $2 \mathrm{~kg}$.

The parameters $a_{0}, a_{1}$ and $b_{0}$ reach their converged state in less than $1 \mathrm{sec}$, proving the efficacy of the RLS-based algorithm. Similar to the performances shown in Fig. 4, the IM-Controller manages set-point tracking in less than $1 \mathrm{sec}$, with a mean steady state error of approximately $0.000137 \mathrm{~m}$.

3) Adaptability: Figures 6 and 7 present the test-PAM response under sinusoidal reference signal at $0.05 \mathrm{~Hz}$ and ranging between 0.02 and $0.07 \mathrm{~m}$, while being under a constant load of $2 \mathrm{~kg}$. Specifically, Fig. 6 displays the displacement $x$, the error signal $e=x_{\text {ref }}-x$ and the velocity $\dot{x}$, whilst Fig. 7 displays the pressure $P$ of the air supplied to the test-PAM and the operating state signal $r$. 

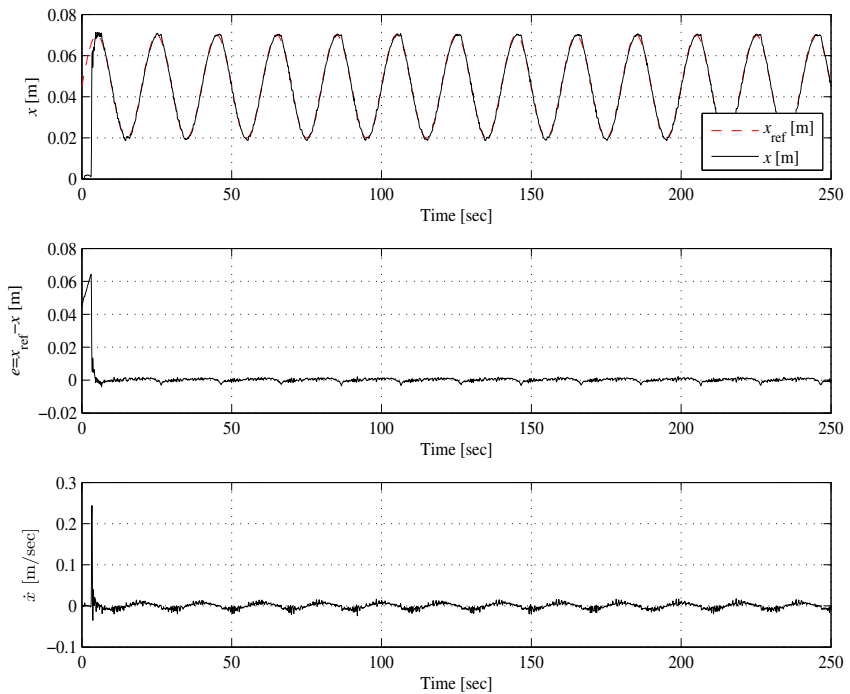

Figure 6. The experimental PAM system response for $x_{\text {initial }}=0$ and sinusoidal reference signal.
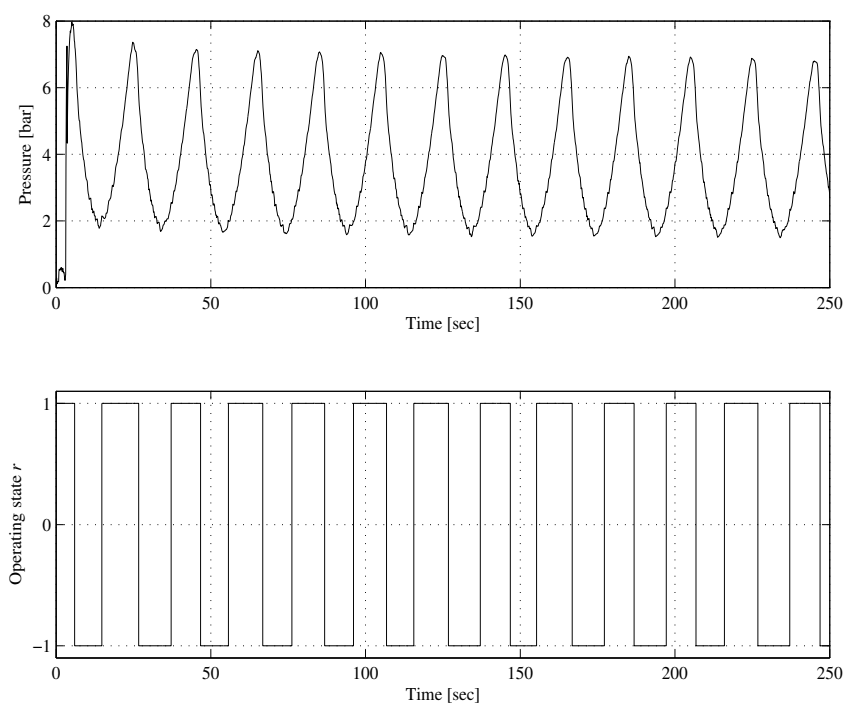

Figure 7. The control effort $P$ and operating state $r$ for the reference signal displayed in Fig. 6.

During the initial 3 seconds of the response, white noise input pressure has been applied to the test-PAM. The goal is to accelerate the identification algorithm and decrease the effect of transient phenomena during the first learning period of the reference signal.

As stated in Section 2, in order to improve the controller transition between inflation and deflation operating states, the recursive identification process restarts whenever a state change occurs. The IM-Controller succeeds in tracking the sinusoidal reference signal while maintaining a mean steady state error $e$ of approximately $0.000173 \mathrm{~m}$.

Observation of the pressure signal $P$ after the first learning period, shows gradual shift towards lesser values over operating time. Specifically, the shift occurs as a decrease of about 0.3bar peak-to-peak after 250sec of response time. This effect concurs with the fact that the viscoelastic properties of the PAM actuator, combined with
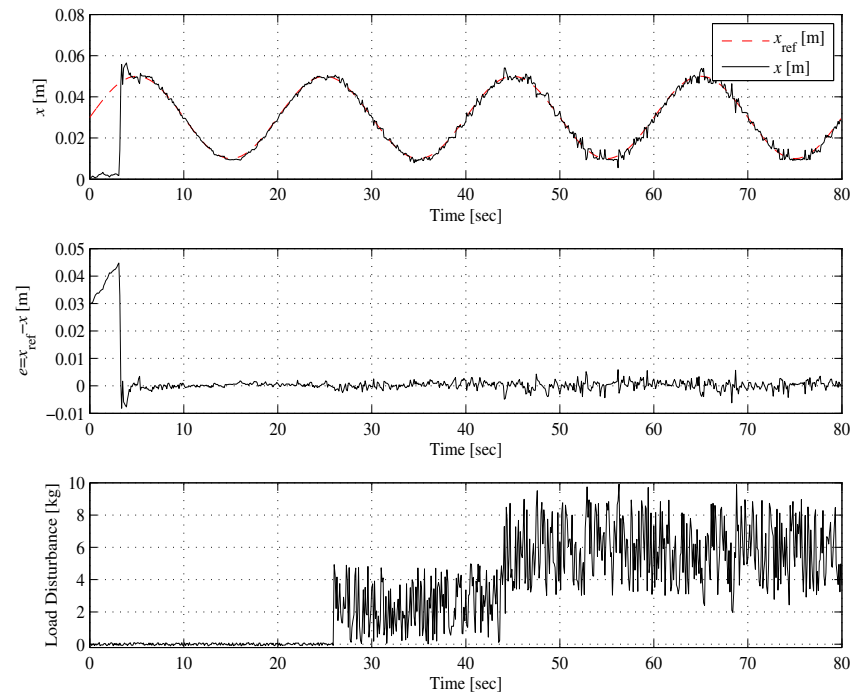

Figure 8. Long-duration and random-amplitude output disturbance rejection of the IMC structure during sinusoidal reference tracking.

the thermal dissipation due to friction phenomena, result in a gradual pressure-displacement hysteresis loop shift [29]. In the currently presented experiment, the recursive algorithm updates the ARMA model, causing the IMC structure to shift the control effort so as to maintain the same displacement outcome.

Thus, the proposed scheme manages to successfully adapt to the alterations in PAM dynamics due to viscoelastic and thermal nonlinear phenomena, which occur and build up over operating time.

4) Robustness: To test the robust behavior of the IMC scheme, the test-PAM is connected to the additional pair of PAMs via a pulley. The additional pair is being utilized as a powerful output disturbance generator in order to further test the robustness of the proposed control structure. Specifically, during sinusoidal reference tracking and at approximately $26 \mathrm{sec}$ of response time, white noise input pressure is supplied to the stretching PAMs. Thus, variable loading ranging between 0 and $5 \mathrm{~kg}$ is provided to the test-PAM, causing a form of long-duration output disturbance. At approximately $44 \mathrm{sec}$, the white noise range is altered, causing intense loading ranging between 3 and $10 \mathrm{~kg}$.

During both phases of this experiment, which are displayed in Fig. 8, the IM-Controller manages to sufficiently reject the additive output disturbance, despite its rapid change rate of amplitude. The test-PAM continues to track the sinusoidal signal despite the output disturbances, further proving the robustness of the IMC scheme.

\section{CONCLUSION}

In this article, the model identification and control problem of a PAM was considered. The identification of the PAM's model parameters was carried out by a Recursive Least Square (RLS) based algorithm, while an Internal Model Control (IMC) structure was synthesized. Experimental studies were utilized to prove the overall efficiency of the suggested control scheme, by evaluating the 
controller's: a) accuracy through selected positioning scenarios, b) adaptability through hysteresis shift compensation and c) robustness through disturbance cancellation.

\section{REFERENCES}

[1] P. Warszawska, "Artificial Pneumatic Muscles," U.S. Patent No. 6704918, 1967.

[2] H. F. Schulte, "The characteristics of the McKibben artificial muscle," in The Application of External Power in Prosthetics and Orthotics, 1961, pp. 94-115.

[3] V. Nickel, J. Perry, and A. Garrett, "Development of useful function in the severely paralyzed hand," in Journal of Bone and Joint Surgery, vol. 45-A, no. 5, pp. 933-952, 1963.

[4] R. H. Gaylord, "Fluid Actuated Motor System and Stroking Device," U.S. Patent No. 9518851958.

[5] J. Yarlott, "Fluid actuator," U.S. Patent No. 3645 173, 1972.

[6] H. Herr and R. Kornbluh, "New horizons for orthotic and prosthetic technology: artificial muscle for ambulation," in Proceedings of SPIEs Smart Structures and Materials, vol. 5385, no. 2004, 2004, pp. $1-9$.

[7] G. Andrikopoulos, G. Nikolakopoulos, and S. Manesis, "A Survey on applications of Pneumatic Artificial Muscles," in Mediterranean Conference on Control and Automation (MED), 2011, pp. 14391446.

[8] T. Hesselroth, K. Sarkar, P. Van der Smagt, and K. Schulten, "Neural network control of a pneumatic robot arm," in IEEE transactions on System Man Cybernetics, vol. 24, no. 1, pp. 28-38, 1994.

[9] G. A. Medrano-Cerda, C. J. Bowler, and D. G. Caldwell, "Adaptive position control of antagonistic pneumatic muscle actuators," in Proceedings 1995 IEEE/RSJ International Conference on Intelligent Robots and Systems. Human Robot Interaction and Cooperative Robots, 1995, vol. 1, pp. 378-383.

[10] D. W. Repperger, K. R. Johnson, and C. A. Phillips, "A VSC position tracking system involving a large scale pneumatic muscle actuator," in Proceedings of the 37th IEEE Conference on Decision and Control (Cat. No.98CH36171), 1998, vol. 4, pp. 4302-4307.

[11] D. W. Repperger, C. A. Phillips, and M. Krier, "Controller design involving gain scheduling for a large scale pneumatic muscle actuator," in Proceedings of the 1999 IEEE International Conference on Control Applications (Cat. No.99CH36328), 1999, vol. 1, pp. 285-290.

[12] X. Chang and J. H. Lilly, "Fuzzy control for pneumatic muscle tracking via evolutionary tuning," in Intelligent Automation and Soft Computing, vol. 9, no. 4, pp. 227-44, 2003.

[13] K. Balasubramanian and K. S. Rattan, "Fuzzy logic control of a pneumatic muscle system using a linearing control scheme," in 22nd International Conference of the North American Fuzzy Information Processing Society, NAFIPS 2003, 2003, pp. 432-436.

[14] D. B. Reynolds, D. W. Repperger, C. A. Phillips, and G. Bandry, "Modeling the Dynamic Characteristics of Pneumatic Muscle," in Annals of Biomedical Engineering, vol. 31, no. 3, pp. 310-317, Mar. 2003.

[15] J. H. Lilly, "Adaptive tracking for pneumatic muscle actuators in bicep and tricep configurations.," in IEEE Transactions on Neural Systems and Rehabilitation Engineering, vol. 11, no. 3, pp. 333-339, Sep. 2003.

[16] S. W. Chan, J. H. Lilly, D. W. Repperger, and J. E. Berlin, "Fuzzy PD+I learning control for a pneumatic muscle," in 12th IEEE International Conference on Fuzzy Systems, 2003. FUZZ '03., 2003, pp. 278-283.

[17] K. K. Ahn and T. D. C. Thanh, "Nonlinear PID control to improve the control performance of the pneumatic artificial muscle manipulator using neural network," in Journal of mechanical science and technology, vol. 19, no. 1, pp. 106-115, 2005.

[18] H. Aschemann and D. Schindele, "Sliding-Mode Control of a HighSpeed Linear Axis Driven by Pneumatic Muscle Actuators," IEEE Transactions on Industrial Electronics, vol. 55, no. 11, pp. 3855-3864, Nov. 2008.
[19] T. Choi and J. Lee, "Control of Manipulator Using Pneumatic Muscles for Enhanced Safety," IEEE Transactions on Industrial Electronics, vol. 57, no. 8, pp. 2815-2825, Aug. 2010.

[20] D. Shin, I. Sardellitti, and O. Khatib, "A Hybrid Actuation Approach for Human-Friendly Robot Design," IEEE International Conference on Robotics and Automation (ICRA), pp. 1747-1752, May 19-23 2008, Pasadena, California, USA.

[21] M. V. Damme, B. Vanderborght, B. Verrelst, R. V. Ham, F. Daerden, and D. Lefeber, "Proxy-based Sliding Mode Control of a Planar Pneumatic Manipulator," The International Journal of Robotics Research (IJRR), vol. 28, pp. 266-284, February 2009.

[22] G. Andrikopoulos, G. Nikolakopoulos, I. Arvanitakis and S. Manesis, "Piecewise affine modeling and constrained optimal control for a pneumatic artificial muscle," to be appeared in IEEE Transactions on Industrial Electronics.

[23] M. T. Tham, "Internal model control," Chemical and Process Eng., University of Newcaslte upon Tyne, 2002.

[24] D. E. Rivera, "Internal model control: a comprehensive view," Dept. of Chemical, Bio and Materials Eng., Arizona State University, Tempe, USA, 1999.

[25] G. Nikolakopoulos, A. Tzes, "Reconfigurable Adaptive Control Based on Lattice Filtering", in Journal of Mathematics and Computers in Simulation, Vol. 60, No. 3-5, pp. 303-314, 2002.

[26] G. Nikolakopoulos, A. Tzes and Y. Koveos, "Application of Adaptive Lattice Filters to Internal Model Control", in International Journal of Adaptive Control and Signal Processing, Vol. 22, No. 6, pp 569-589, September 2007.

[27] K. J. Astrom, and B. Wittenmark, "Adaptive control", 2nd edition, Addison-Wesley, 1974.

[28] C. E. Garcia, and M. Morari. "Internal Model Control -1. A Unifying Review and Some New Results." Ind. Eng. Chem. Process Des. \& Dev. 21, 308-323, 1982.

[29] G. Andrikopoulos, G. Nikolakopoulos and S. Manesis, "An Experimental Study on Thermodynamic Properties of Pneumatic Artificial Muscles", in 20th Mediterranean Conference on Control and Automation (MED), 3-6 July 2012, Barcelona, Spain. 